\title{
Self-similar and charged spheres in the diffusion approximation
}

\author{
W. Barreto and A. Da Silva \\ Laboratorio de Física Teórica, Departamento de Física, \\ Escuela de Ciencias, Núcleo de Sucre, \\ Universidad de Oriente, Cumaná, Venezuela.
}

October 27, 2018

\begin{abstract}
We study spherical, charged and self-similar distributions of matter in the diffusion approximation. We propose a simple, dynamic but physically meaningful solution. For such a solution we obtain a model in which the distribution becomes static and changes to dust. The collapse is halted with damped mass oscillations about the absolute value of the total charge.
\end{abstract}

\section{Introduction}

Several authors have considered charged distributions of matter [1, 2, 3, 4, 5, 6, 7, 8, 9, 10, 11, although it is well known that astrophysical objects are not significantly charged. Nevertheless, in some stages of the gravitational collapse even a small amount of charge can change the final state of the body. Some interesting features of charged collapsing matter justify any effort to get physical insight by studying this problem. For instance, naked singularities can be prevented [12]; the final geometrical structure left over after the complete collapse of a spherically symmetric charged source and of a chargeless rotating star are similar [13] Cauchy horizons, gravitational repulsion and perhaps traversable wormholes are also possible [8].

If the mathematical treatment is simplified, the evolution of a charged distribution of matter can be followed by the Einstein-Maxwell equations. In this paper we explore the self-similar gravitational collapse of charged spheres in the diffusion approximation. It is well known that dissipation due to the emission of massless particles is a characteristic process in the evolution of massive stars. The only plausible mechanism to carry away the bulk of binding energy of the collapsing star, leading to a neutron star or black hole, is neutrino emission 14. It seems clear that the free-streaming process is associated with the initial stages of the collapse, while the diffusion approximation becomes valid toward 
the final stages. The junction conditions at the boundary of a charged and dissipative sphere have been considered with outgoing heat flow and radiation flux [15, 16. On the other hand, the field equations admit homothetic motion [17, 18, 19, 20. Applications of homothetic similarity range from modeling black holes to producing counterexamples to the cosmic censorship conjecture 21, 22, 23, 24, 25, 26, 27, 28. In particular, homothetic charged and isotropic (or anisotropic) fluids have been studied [29, 30, 7].

We observe in the literature that much work has been done under static conditions and dusty charged matter. Also authors make additional assumptions such as equations of state or relationships between metric variables 30, 31. In this paper we obtain a dynamical model from a simple solution to the homothetic motion, without any additional supposition except spherical symmetry and selfsimilarity. In section 2 we write the field equations and the junction conditions. The equations at the surface of the distribution of matter are presented in section 3. In section 4 we write the symmetry equations to describe self-similarity. In section 5, we show an example from a simple solution which we discuss in the last section.

\section{$2 \quad$ Field equations and matching}

Let us consider a non-static distribution of matter which is spherically symmetric and consists of charged fluid of energy density $\rho$, pressure $p$, electric charge density $\sigma$ and radiation energy flux $q$ diffusing in the radial direction, as measured by a local Minkowskian observer comoving with the fluid. In radiation coordinates 32 the metric takes the form

$$
d s^{2}=e^{2 \beta}\left(\frac{V}{r} d u^{2}+2 d u d r\right)-r^{2}\left(d \theta^{2}+\sin \theta^{2} d \phi^{2}\right),
$$

where $\beta$ and $V$ are functions of $u$ and $r$. Here $u$ is the time-like coordinate: in the flat space-time $u$ is just the retarded time, so that surfaces of constant $u$ represent null cones open to the future; $r$ is a null coordinate $\left(g_{r r}=0\right)$ such that surfaces of constant $r$ and $u$ are spheres; $\theta$ and $\phi$ are the usual angular coordinates. Also, in this paper we use relativistic units $(G=c=1)$.

The Einstein field equations, $\mathbf{G}=-8 \pi \mathbf{T}$, are considered with the energymomentum tensor $\mathbf{T}=(\rho+p) \mathbf{v} \otimes \mathbf{v}-p \mathbf{g}+\mathbf{q} \otimes \mathbf{v}+\mathbf{v} \otimes \mathbf{q}+\mathbf{E}$, where $\mathbf{v}$ and $\mathbf{q}$ are the four-velocity and the heat flux four-vector, respectively, which must be orthogonal; $\mathbf{E}$ is the electromagnetic field energy-momentum tensor constructed with the Maxwell field tensor $\mathbf{F}$ as is usually done. The Maxwell field equations, $\mathbf{d}^{*} \mathbf{F}=4 \pi^{*} \mathbf{J}$ and $\mathbf{d F}=0$, are coupled minimally with gravitation, where $\mathbf{J}=\sigma \mathbf{v}$ is the electric current four-vector.

Because of the spherical symmetry, only the radial electric field $F^{u r}=-F^{r u}$ is non-vanishing. If we define the function $C(u, r)$ by the relation

$$
F^{u r}=C e^{-2 \beta} / r^{2},
$$


the inhomogeneous Maxwell equations become

$$
C_{, r}=4 \pi r^{2} J^{u} e^{2 \beta}
$$

and

$$
C_{, u}=-4 \pi r^{2} J^{r} e^{2 \beta}
$$

where the comma subscript represents partial derivative with respect to the indicated coordinate. The function $C(u, r)$ is naturally interpreted as the charge within the radius $r$ at time $u$. Thus, the inhomogeneous field equations entail the conservation of charge inside a sphere comoving with the fluid, expressed by

$$
\mathbf{v}(C)=0 .
$$

Let $\omega$ be the velocity of matter as seen by a Minkowskian observer moving at $-\omega$ with respect to the local comoving frame; the matter velocity in radiation coordinates is then given by

$$
\frac{d r}{d u}=\frac{V}{r} \frac{\omega}{1-\omega}
$$

Introducing the mass function by

$$
V=e^{2 \beta}\left(r-2 \tilde{m}(u, r)+C^{2} / r\right),
$$

we can write the Einstein equations as

$$
\begin{gathered}
\frac{\rho+p \omega^{2}}{1-\omega^{2}}+\frac{2 \omega q}{1-\omega^{2}}=\frac{e^{-2 \beta}\left(C C_{, u} / r-\tilde{m}_{, u}\right)}{4 \pi r\left(r-2 \tilde{m}+C^{2} / r\right)}+\frac{\tilde{m}_{, r}-C C_{, r} / r}{4 \pi r^{2}}, \\
\frac{\rho-p \omega}{1+\omega}-\left(\frac{1-\omega}{1+\omega}\right) q=\frac{\tilde{m}_{, r}-C C_{, r} / r}{4 \pi r^{2}}, \\
\left(\frac{1-\omega}{1+\omega}\right)(\rho+p)-2\left(\frac{1-\omega}{1+\omega}\right) q=\frac{\beta_{, r}}{2 \pi r^{2}}\left(r-2 \tilde{m}+C^{2} / r\right), \\
p=-\frac{1}{4 \pi} \beta_{, u r} e^{-2 \beta}+\frac{1}{8 \pi}\left(1-2 \tilde{m} / r+C^{2} / r^{2}\right)\left(2 \beta_{, r r}+4 \beta_{, r}^{2}-\beta_{, r} / r\right) \\
+\frac{1}{8 \pi r}\left[3 \beta_{, r}\left(1-2 \tilde{m}_{, r}\right)-\tilde{m}_{, r r}\right]+\frac{3 \beta_{, r}}{8 \pi r}\left(2 C C_{, r} / r-C^{2} / r^{2}\right) \\
+\frac{1}{8 \pi r^{2}}\left(C_{, r}^{2}+C C_{, r r}-2 C C_{, r} / r\right) .
\end{gathered}
$$

We describe the exterior space-time by the Reissner-Nordström-Vaidya metric

$$
d s_{+}^{2}=\left(1-\frac{2 m(u)}{r}+\frac{C_{T}^{2}}{r^{2}}\right) d u^{2}+2 d u d r-r^{2}\left(d \theta^{2}+\sin ^{2} \theta d \phi^{2}\right),
$$

where $m(u)$ is the total mass and $C_{T}$ the total charge. It can be shown that the junction conditions to match the metrics (1) and (12), across the moving 
boundary surface $r=a(u)$, are equivalent to the continuity of the functions $\tilde{m}(u, r)$ and $\beta(u, r)$ across the boundary, that is, $\tilde{m}(u, a)=m(u)$ and $\beta(u, a)=$ 0 , and to the equation

$$
-\beta_{, u a}+\left(1-\frac{2 m}{a}+\frac{C_{T}^{2}}{a^{2}}\right) \beta_{, r a}-\frac{\tilde{m}_{, r a}}{2 a}+\frac{C_{T} C_{, r a}}{2 a^{2}}=0,
$$

where the subscript $a$ indicates that the quantity is evaluated at the surface $r=a(u)$. We have used the continuity of the radial electric field through the boundary assuming no surface free charge density, yielding $C(u, a)=C_{T}$. It should be mentioned that the discontinuity of the pressure at the boundary, $p_{a}=q_{a}$, is a direct consequence of (6), (9), (10) and the junction conditions [33, 15, 16].

\section{The surface equations}

One of the surface equations is just (6) evaluated at $r=a$, which takes the form

$$
\dot{a}=\left(1-\frac{2 m}{a}+\frac{C_{T}^{2}}{a^{2}}\right) \frac{\omega_{a}}{1-\omega_{a}},
$$

where (7) has been used as well as the junction conditions for $\tilde{\mathrm{m}}, \beta$ and $C$. It is convenient to scale the variables by the initial mass $m(0)$, such that

$$
A \equiv \frac{a}{m(0)} ; M \equiv \frac{m}{m(0)} ; \frac{u}{m(0)} \rightarrow u ; \frac{C_{T}}{m(0)} \rightarrow C_{T} .
$$

Defining the surface potential $\mathrm{F}$ and $\Omega$ by

$$
F \equiv 1-\frac{2 M}{A}+\frac{C_{T}^{2}}{A^{2}}
$$

and

$$
\Omega \equiv \frac{1}{1-\omega_{a}},
$$

(14) can be written in the form

$$
\dot{A}=F(\Omega-1),
$$

which is the first surface equation.

The second surface equation relates the total mass loss rate to the energy flux through the boundary surface. It has been shown 33] that this equation can be written as

$$
\dot{F}=\frac{2 Q F+(1-F) \dot{A}}{A}-\frac{C_{T}^{2} \dot{A}}{A^{3}},
$$

where

$$
Q=(2 \Omega-1)\left(4 \pi r^{2} q\right)_{r=a}
$$


The third surface equation is the charge conservation law given by (5), evaluated at the boundary

$$
C_{, u a}=-\dot{A} C_{, r a} .
$$

The fourth surface equation is model dependent. This correspond to the Bianchi identity $T_{r, \mu}^{\mu}=0$, given by

$$
\begin{gathered}
\frac{\partial \tilde{p}}{\partial r}+\frac{\tilde{\rho}+\tilde{p}}{\left(1-2 \tilde{m} / r+C^{2} / r^{2}\right)}(4 \\
\left.\qquad r \tilde{p}+\frac{\tilde{m}}{r^{2}}-\frac{C^{2}}{r^{3}}\right)-e^{-2 \beta}\left(\frac{\tilde{\rho}+\tilde{p}}{1-2 \tilde{m} / r+C^{2} / r^{2}}\right)_{, u} \\
=\frac{2}{r}(p-\tilde{p})+\frac{C C_{, r}}{4 \pi r^{4}}
\end{gathered}
$$

where $\tilde{p}=(p-\omega \rho) /(1+\omega)-(1-\omega) q /(1+\omega)$ and $\tilde{\rho}=(\rho-\omega p) /(1+\omega)-$ $(1-\omega) q /(1+\omega)$. Equation (21) is the generalization of that of TolmanOppenheimer-Volkov for non-static and charged radiative situations [33] (see Reference [4] for the static case).

\section{Self-similar space-time}

Self-similarity is invariably defined by the existence of a homothetic Killing vector field [18. We shall assume that the spherical distribution admits a oneparameter group of homothetic motions. A homothetic vector field on the manifold is one that satisfies $£_{\xi} g=2 n g$ on a local chart, where $n$ is a constant on the manifold and $£$ denotes the Lie derivative operator. If $n \neq 0$ we have a proper homothetic vector field and it can always be scaled so as to have $n=1$; if $n=0$ then $\xi$ is a Killing vector on the manifold 34 35. So, for a constant rescaling, $\xi$ satisfies

$$
£_{\xi} \mathbf{g}=2 \mathbf{g}
$$

and has the form

$$
\xi=\Lambda(u, r) \partial_{u}+\lambda(u, r) \partial_{r} .
$$

The homothetic equations reduce to

$$
\begin{gathered}
\xi(X)=Z \xi(Z), \\
\xi(Y)=0,
\end{gathered}
$$

where $\lambda=r, \Lambda=\Lambda(u), X=\tilde{m} / r, Y=\Lambda e^{2 \beta} / r$ and $Z=C / r$. Therefore, $X=X(\zeta), Y=Y(\zeta)$ and $Z=Z(\zeta)$ are solutions if the self-similar variable is defined as

$$
\zeta \equiv r e^{-\int d u / \Lambda} .
$$

In the next section we propose a simple solution which evolves toward staticity, rendering an inhomogeneous and dusty fluid sphere. 


\section{A simple example}

The simple solution $\tilde{m}=m r / a, e^{2 \beta}=r / a$ and $C=C_{T} r / a$ satisfies the additional symmetry equations (24) and (25), the charge conservation equation (20), the continuity of the radial electric field and the continuity of the first fundamental form. Now, with these solutions and the junction condition (13) (continuity of the second fundamental form), $\Omega$ is determined by

$$
\Omega=\frac{1-F-C_{T}^{2} / A^{2}}{2 F} .
$$

The heat flow at the surface is obtained from the conservation equation (21) evaluated at the surface, resulting in

$$
Q=\frac{F-C_{T}^{2} / A^{2}}{2 F}\left(1-2 F-C_{T}^{2} / A^{2}\right)
$$

This last equation, together with (27), must be taken into account to integrate numerically the equations (17) and (18). Using a standard Runge-Kutta algorithm and the initial conditions $A(0)=3.50$ and $m(0)=1.00$, we study the effect of charge on collapse. Once the boundary evolution and its energetic are determined, we calculate the physical variables from the field equations. Figures 1-7 show the results obtained for our simple solution. We shall discuss them in the next section.

\section{Discussion}

Figure 1 displays the evolution of the surface for different values of the total charge. It is clear that the increase of the total charge favors the collapse in a first stage of the evolution. Later the collapse is halted, with damped oscillations rendering a distribution which is less compact for greater total charge. We show in Figure 2 how the Bondi mass decreases and oscillates about the total charge (given positive) until reaching the same value of $C_{T}$. Figures $3-6$ sketch the pressure $p$, the density $\rho$, the heat flow $q$ and the matter velocity $d r / d u$. Observe that the whole sphere of fluid becomes dust ( $p=0$ at all points) and inhomogeneous when staticity is reached. It is interesting to note that the cooling proceeds with emission and absorption of energy and consequently all the shells bounce and contract as a unit, over and over, until reaching staticity in the whole sphere. Also it is striking how the ratio $p / \rho$ is a function only of the Bondi time, i.e., it is the same at any point of the charged distribution (see Figure 7).

We would like to stress that our solution, in spite of its simplicity, behaves very well when one takes into account the results reported by other authors. The sphere collapses and rebounds as a unit [13, over and over, until reaching staticity. Equilibrium configurations are possible, with no necessity of internal pressure; moreover, electric charge halts the gravitational collapse [1, 4]. The spheres of charged matter can oscillate and the final static configuration is 
reached when the total mass is equal to the total charge [2, that is, the charged spheres of dust in equilibrium belong to the interior Papapetrou-Majumdar class 6].

Finally, we make special mention of the Herrera and Ponce de León homothetic models [7. For a null radial pressure at the surface (as a boundary condition) they find a charged dust. Otherwise, the distribution is infinitely extended. From an altogether physical point of view, perhaps it is possible to find finite perfect (and self-similar) fluid sources with zero pressure at the surface, considering free-streaming as the inner transport mechamism. In general, Herrera and Ponce de León find that the total charge is less than the total mass. The equality will only be valid in the case of homothetic charged dust spheres.

\section{Acknowledgments}

We benefited from research support by the Consejo de Investigación under Grant CI-5-1001-0774/96 of the Universidad de Oriente. We would like to thank Loren Lockwood for his helpful reading and valuable comments.

\section{References}

\section{References}

[1] Bonnor W B 1965 Mon. Not. Royal Astr. Soc. 129443

[2] Faulkes M C 1969 Can. J. Phys. 471989

[3] Shvarsman V F 1971 Sov. Phys. JETP 33475

[4] Bekenstein J D 1971 Phys. Rev. D4 2185

[5] Bonnor W B 1975 Mon. Not. Royal Astr. Soc. 170643

[6] Cooperstock F I and de la Cruz V 1978 Gen. Rel. Grav. 9835

[7] Herrera L and Ponce de León J 1985 J. Math. Phys. 262302

[8] Ori A 1990 Class. Quantum Grav. 7985

[9] Lake K and Zannias T 1991 Phys. Rev. D 431798

[10] Ori A 1991 Phys. Rev. D 442278

[11] Fayos F, Martin-Prats M M and Senovilla J M M 1995 Class. Quantum Grav. 122565

[12] Ponce de León J 1993 Gen. Rel. Grav 251123

[13] López C 1995 Gen. Rel. Grav. 2785 
[14] Kazanas D and Schramm D 1979 Sources of gravitational Radiation (Cambridge: Cambridge University Press)

[15] de Oliveira A K G and Santos N O 1987 Ap. J 312640

[16] Banerjee A and Dutta Choudhury S B 1989 Gen. Rel. Grav. 21785

[17] Cahill M E and Taub A H 1971 Commun. Math. Phys. 211

[18] Ori A and Piran T 1990 Phys. Rev. D 421068

[19] Henriksen R Patel K 1991 Gen. Rel. Grav. 23527

[20] Lake K and Zannias T 1990 Phys. Rev. D 413866

[21] Carr J and Hawking S 1974 Mon. Not. Royal Astr. Soc. 168399

[22] Bicknell G V and Henriksen N R 1978 Ap. J 2191043

[23] Bicknell G V and Henriksen N R 1978 Ap. J. 225237

[24] Eardley D M, Isemberg J, Mardsden J and Moncrief V 1986 Comm. Math Phys. 106137

[25] Carr J and Yahil A 1990 Ap. J 360330

[26] Lake K 1992 Phys. Rev Lett. 683129

[27] Brady P R 1995 Phys. Rev. D 514198

[28] Carr B J and Coley A A 1998 gr-qc/9806048

[29] Pant D N and Sah A 1979 J. Math. Phys. 202537

[30] Tikekar R 1984 J. Math. Phys. 251481

[31] Humi M and Mansour J 1984 Phys. Rev. D 291076

[32] Bondi H 1964 Proc. R. Soc. London A 28139

[33] Barreto W and Da Silva A 1996 Gen. Rel. Grav. 28735

[34] Hall G S 1988 Gen. Rel. Grav. 20671

[35] Carot J, Mas L and Sintes A M 1994 J. Math. Phys. 353560 


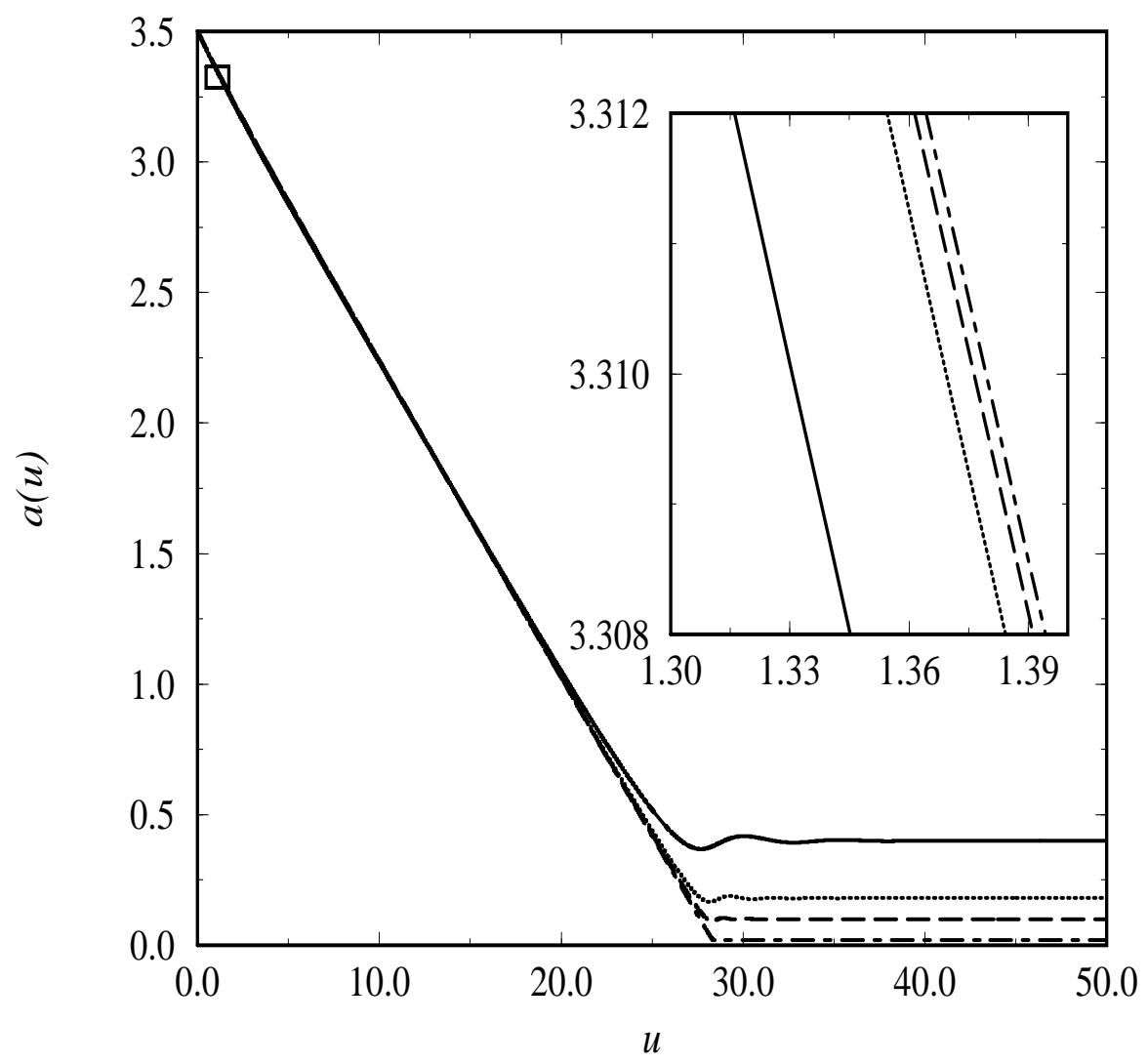

Figure 1: Radius $a$ as a function of the time $u$ for $A(0)=3.5, m(0)=1$ and for different values of the total charge $C_{T}: 0.01$ (dot-dashed line); 0.05 (dashed line); 0.09 (dotted line); 0.2 (solid line). 


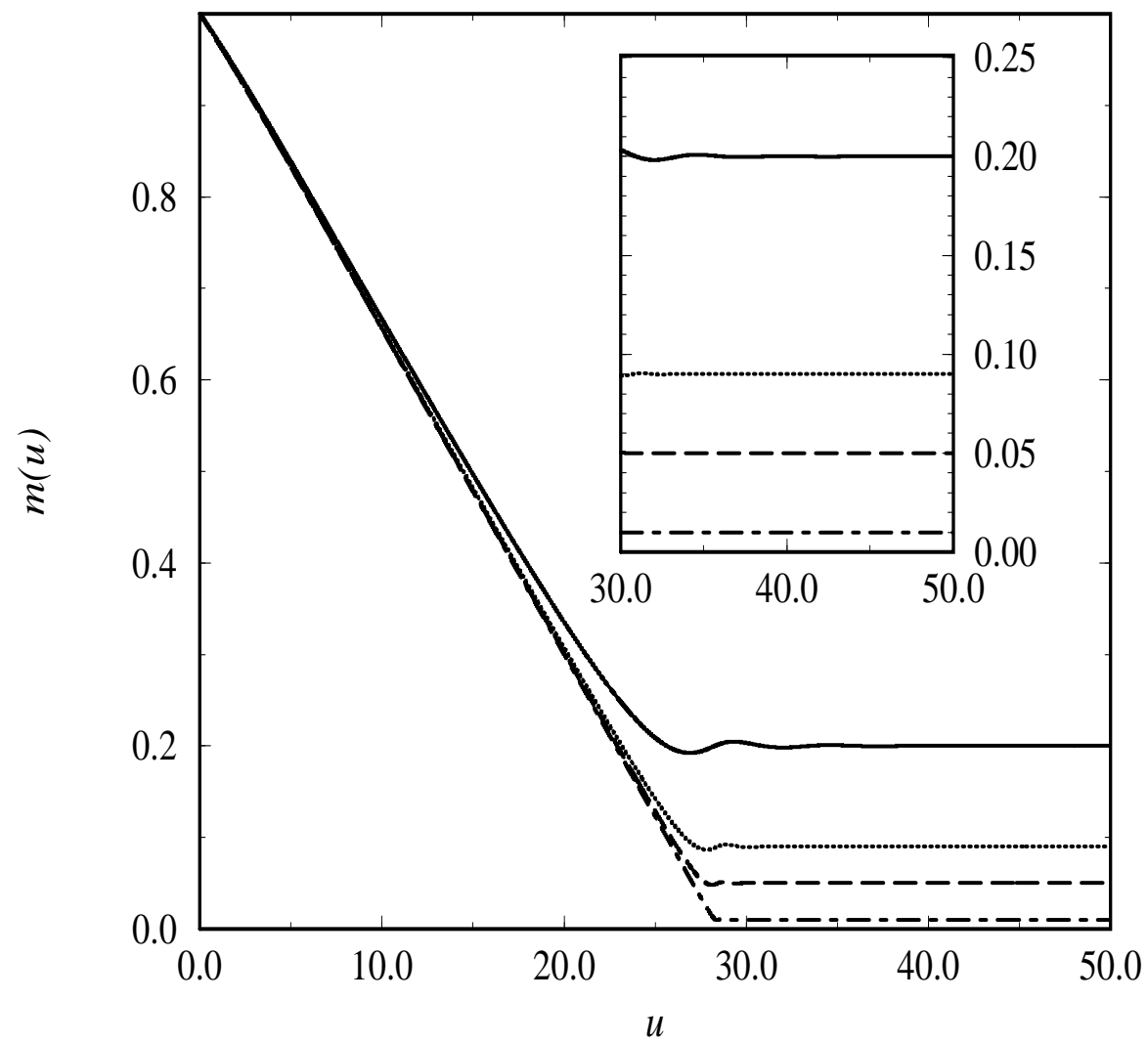

Figure 2: Bondi mass at the surface $m$ as a function of time $u$ for $A(0)=3.5$, $m(0)=1$ and for different values of the total charge $C_{T}$ : 0.01 (dot-dashed line); 0.05 (dashed line); 0.09 (dotted line); 0.2 (solid line). Observe that the final mass in each case is equal to the total charge. 


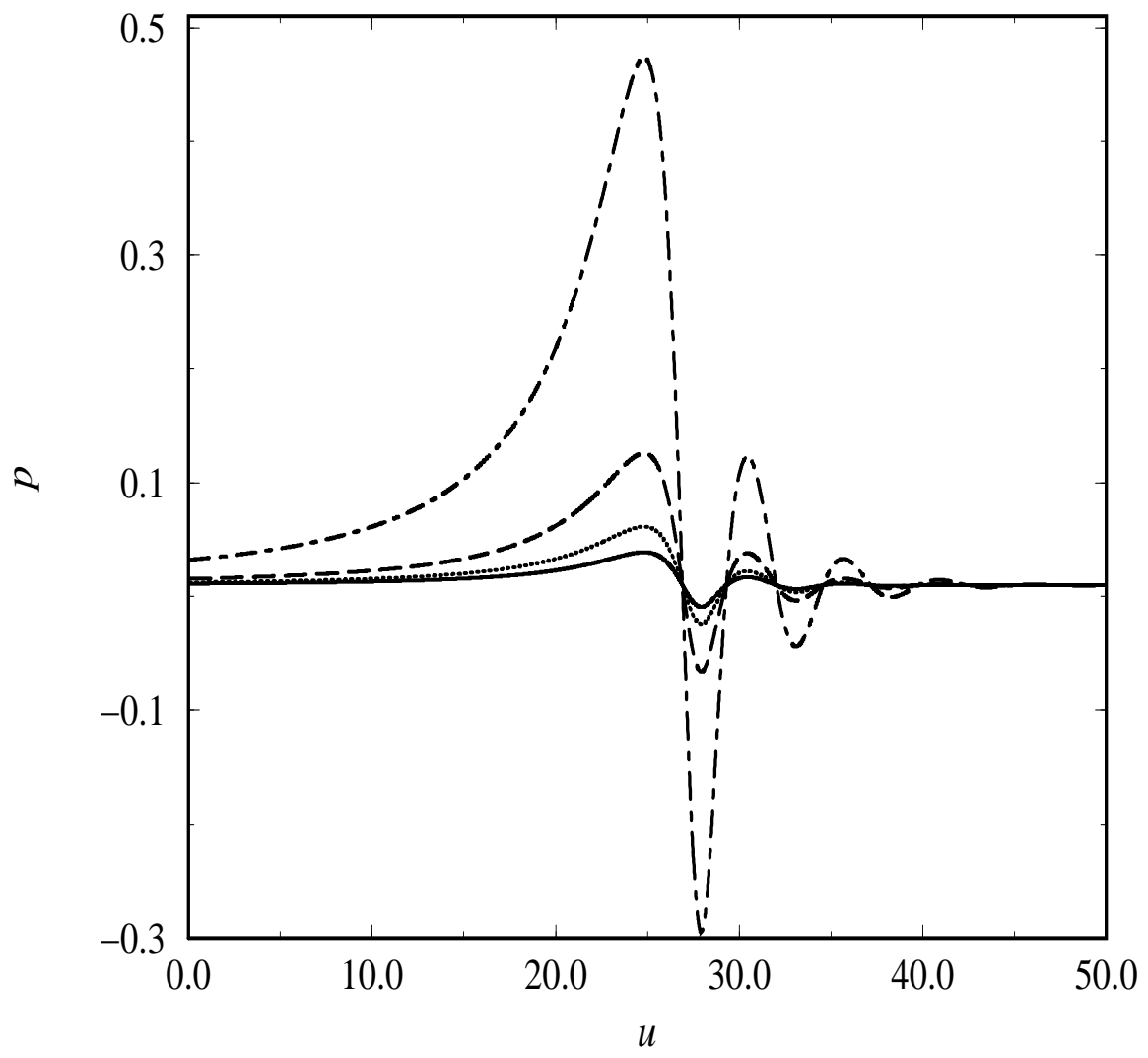

Figure 3: Pressure $p$ as a function of time $u$ for $A(0)=3.5, m(0)=1$ and $C_{T}=0.09$ at different points: $r / a=0.25$ (dot-dashed line); $r / a=0.33$ (dashed line); $r / a=0.50$ (dotted line); $\mathrm{r} / \mathrm{a}=1.00$ (solid line). 


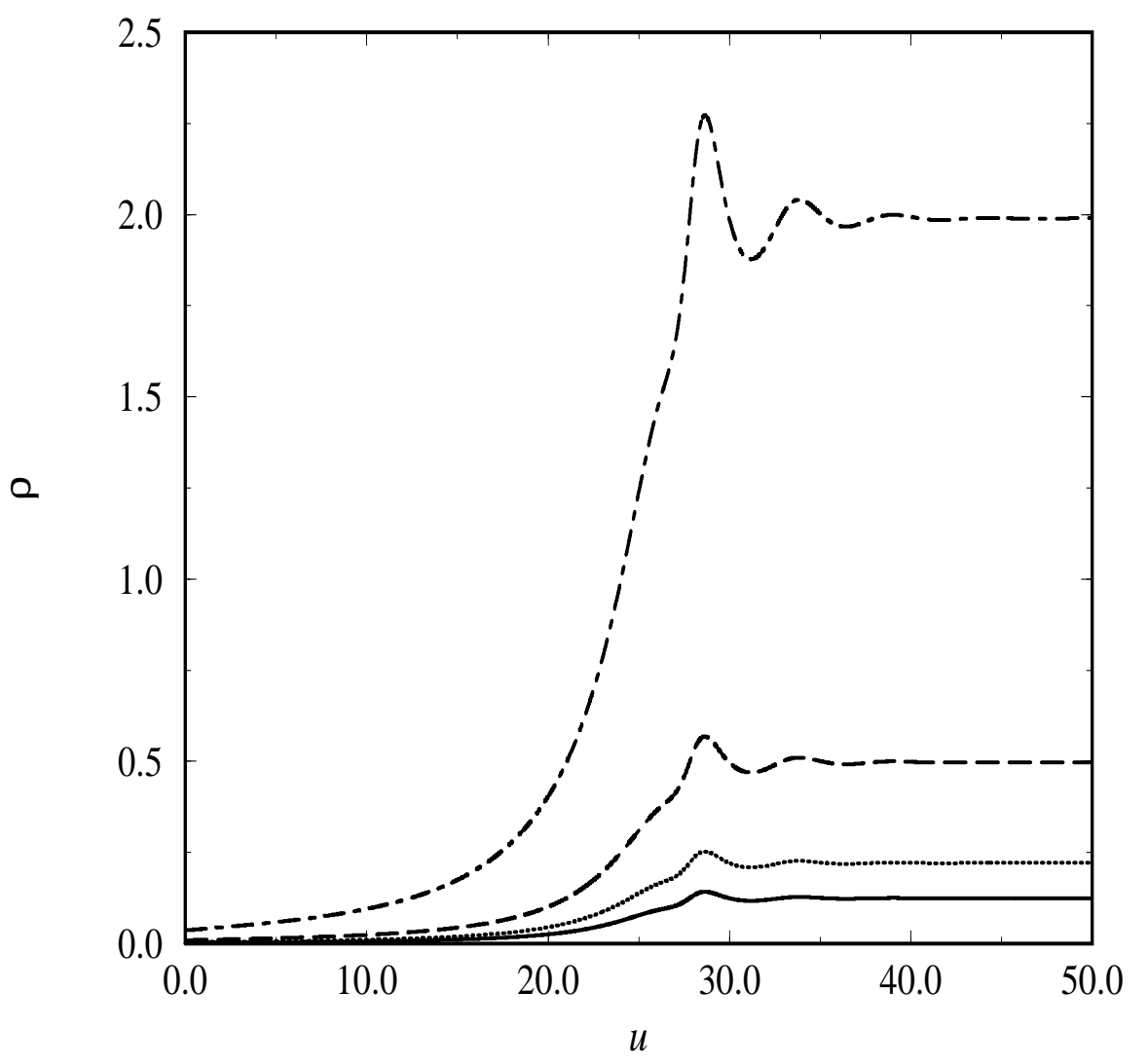

Figure 4: Density $\rho$ as a function of time $u$ for $A(0)=3.5, m(0)=1$ and $C_{T}=0.09$ at different points: $r / a=0.25$ (dot-dashed line); $r / a=0.33$ (dashed line); $r / a=0.50$ (dotted line); $\mathrm{r} / \mathrm{a}=1.00$ (solid line). 


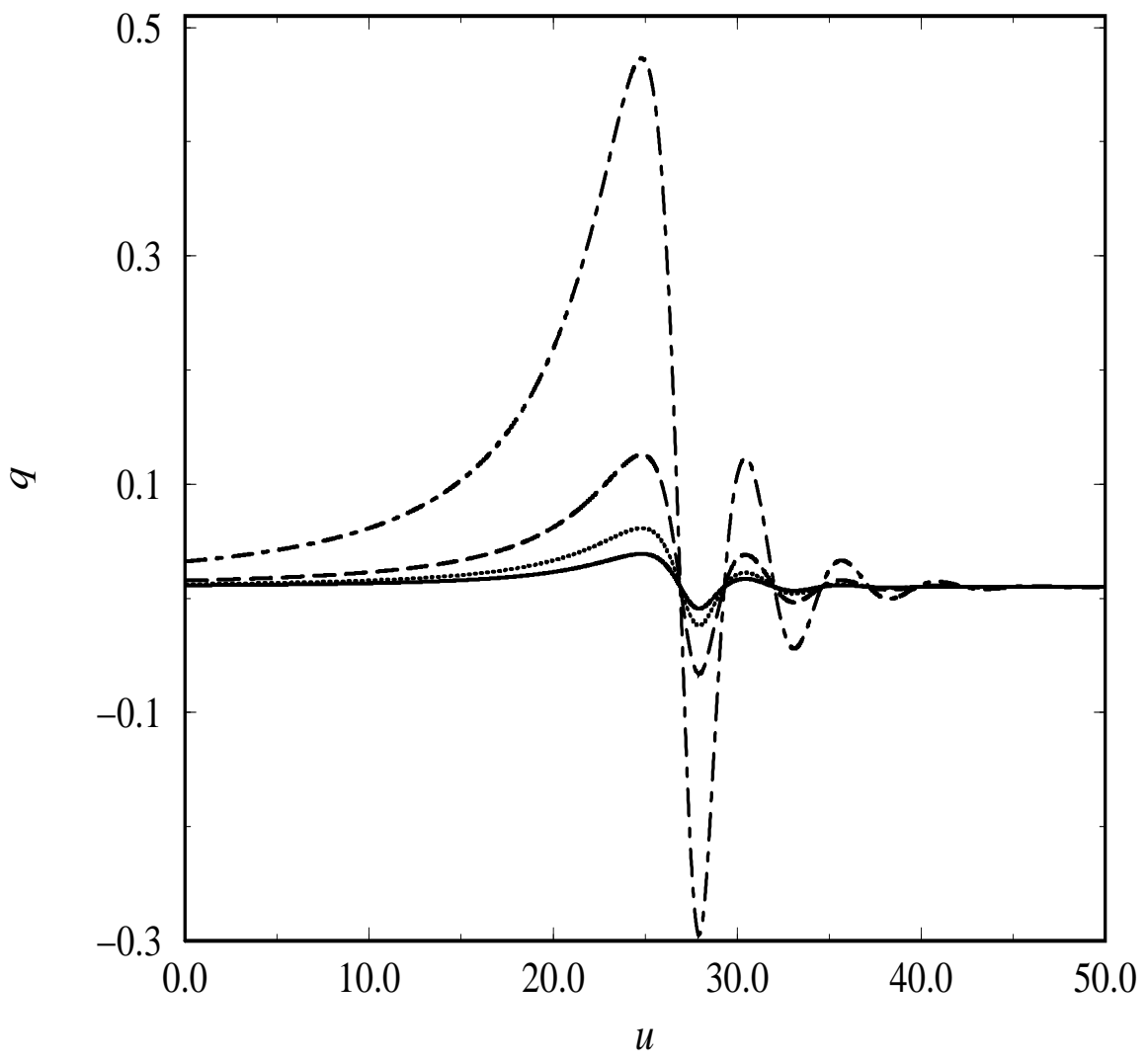

Figure 5: Heat flow $q$ as a function of time $u$ for $A(0)=3.5, m(0)=1$ and $C_{T}=0.09$ at different points: $r / a=0.25$ (dot-dashed line); $r / a=0.33$ (dashed line); $r / a=0.50$ (dotted line); $\mathrm{r} / \mathrm{a}=1.00$ (solid line). 


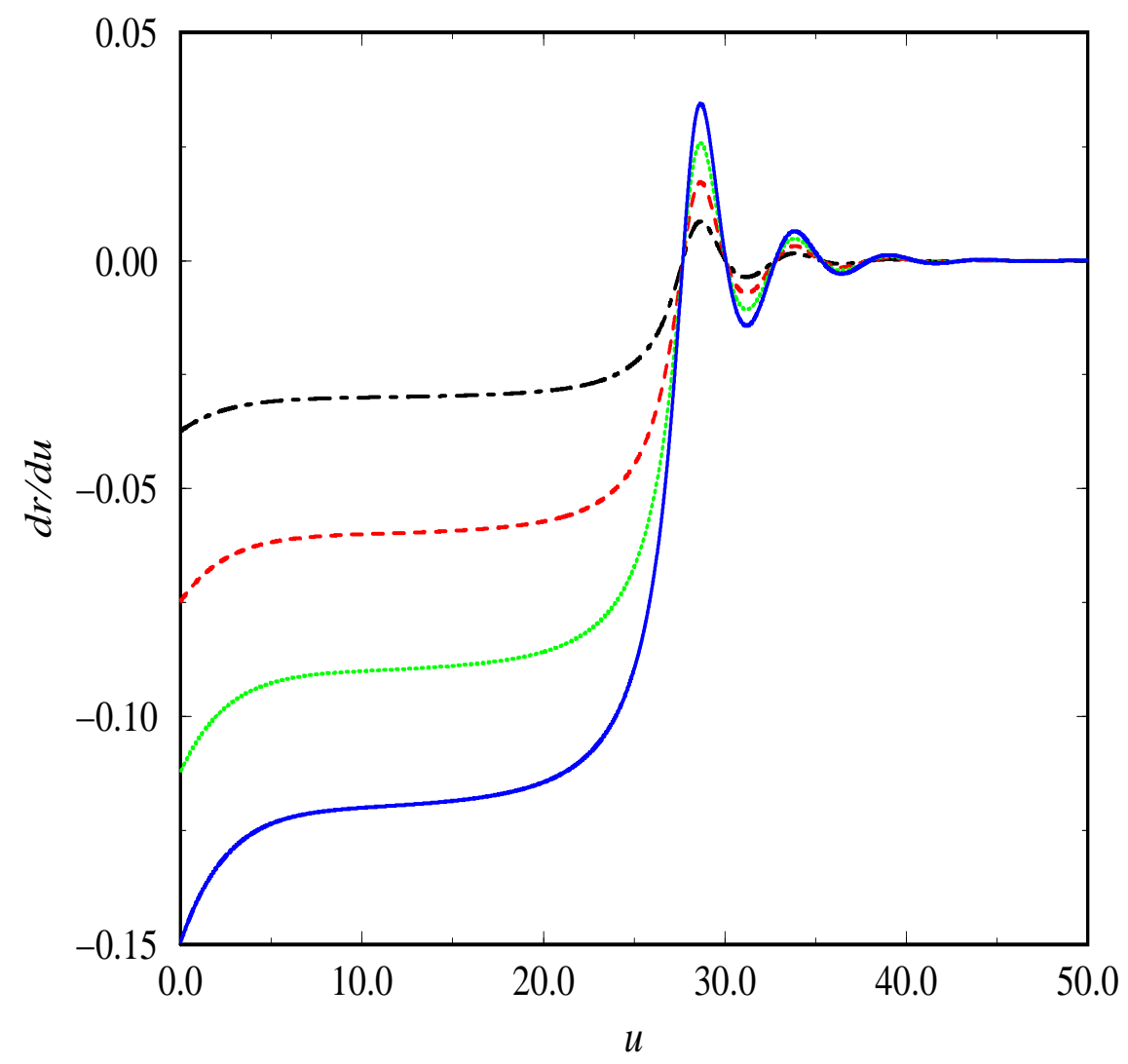

Figure 6: Matter velocity $d r / d u$ as a function of time $u$ for $A(0)=3.5, m(0)=1$ and $C_{T}=0.09$ at different points: $r / a=0.25$ (dot-dashed line) $r / a=0.33$ (dashed line); $r / a=0.50$ (dotted line); $\mathrm{r} / \mathrm{a}=1.00$ (solid line). 


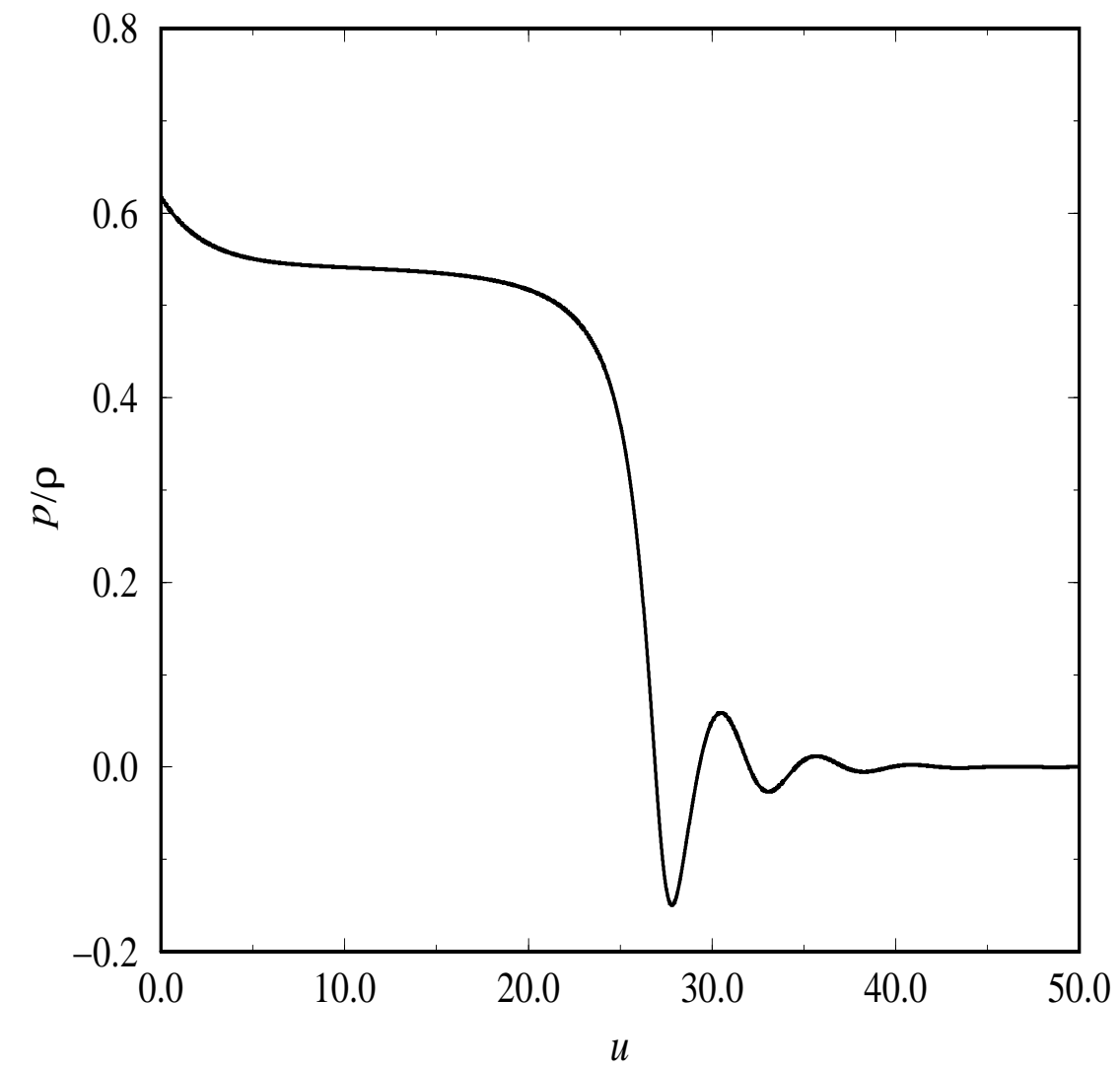

Figure 7: Ratio $p / \rho$ as a function of time $u$ for $A(0)=3.5, m(0)=1$ and $C_{T}=0.09$ at all points. 